\title{
Educación Continua de la UNAE: Un modelo que aporta a la Transformación Educativa del Ecuador (Estudios sobre Educación)
}

Autores: Oscar Antonio Martínez Molina Universidad Nacional de Educación, UNAE oscar.martinez@unae.edu.ec Cuenca, Ecuador Madelin Rodríguez Rensoli Universidad Nacional de Educación, UNAE madelin.rodriguez@unae.edu.ec Cuenca, Ecuador Wilfredo García Felipe Universidad Nacional de Educación, UNAE wilfredo.garcia@unae.edu.ec Cuenca, Ecuador

\section{Resumen}

En este trabajo se presenta el Modelo que aporta a la transformación educativa del Ecuador: la Educación Continua de la Universidad Nacional de Educación, diseñado con el objetivo de satisfacer las necesidades de los docentes a partir de estrategias formativas dirigidas al mejoramiento y transformación de la educación desde la reflexión de su práctica pedagógica, desde un enfoque cooperativo y colaborativo. Se realizó una investigación acción participativa que tienen el objetivo de mejorar y aprender de la propia experiencia desde la reflexión - acción. Finalmente, se incluyen fases para el funcionamiento de la Educación Continua con la sociedad.

Palabras clave: educación continua; investigación; docente; modelo educacional. 


\title{
Continuing Education of the UNAE: A model that contribute to the Educational Transformation of Ecuador (Studies on Education)
}

\begin{abstract}
This paper presents the Model that contributes to the educational transformation of Ecuador: Continuing Education of the National University of Education, designed with the objective of satisfying the needs of teachers based on training strategies aimed at the improvement and transformation of education from the reflection of their pedagogical practice, from a cooperative and collaborative approach. Participatory action research was carried out with the objective of improving and learning from one 's experience from reflection - action. Finally, phases for the operation of Continuing Education with society are included.
\end{abstract}

Keywords: continuing education; research; teacher; educational model. 
"Es preciso no trasmitir la idea (...) que cambiar es fácil; cambiar es difícil, pero posible. Debemos insistir sobre la posibilidad de cambiar, a pesar de las dificultades. La cuestión está en cómo transformar las dificultades en posibilidades. Por esto, en la lucha por cambiar, no podemos ser ni sólo pacientes ni sólo impacientes, sino pacientemente impacientes"

Paulo Freire (1997)

\section{Introducción}

La sociedad contemporánea se enfrenta día a día a un vertiginoso avance científico - tecnológico, que demanda a la Educación Superior nuevos desafíos dirigidos a la implementación de nuevos métodos que favorezcan la transformación y actualización profesional pedagógica del docente. En consecuencia, la educación exige ser concebida desde un enfoque críticoreflexivo en la unidad de lo cognitivo - afectivo, para lograr una comprensión y actuación acorde a las transformaciones sociales de cada contexto. Por ende, lograr un equilibrio en la relación universidad - instituciones escolares, desde valores trascendentales como la igualdad, equidad, solidaridad y el respeto a la diversidad constituye para la educación una prioridad.

El Estado ecuatoriano asume, como una de sus principales responsabilidades y metas, la transformación del sistema educativo en todos los niveles, hasta alcanzar los estándares de eficiencia y calidad que hagan de la educación un verdadero instrumento de equidad, desarrollo sostenible y calidad de vida para todos los ciudadanos del país. La política pública en materia de educación se describe en el Plan Nacional para el Buen Vivir y en el Plan Decenal de Educación 2006-2015, la Ley Orgánica de Educación Superior, Modelo general para la evaluación de carreras con fines de acreditación emitido por el Consejo de Evaluación, Acreditación y Aseguramiento de la Calidad de la Educación Superior (CEAACES). La Universidad Nacional de Educación (UNAE), se corresponde con el desarrollo 
de estas políticas educativas.

Los preceptos establecidos en la Constitución de la República y Plan Nacional de Desarrollo, están dirigidos a: "Fortalecer la educación superior con visión científica y humanística, articulada a los objetivos del Buen Vivir". Al respecto la universidad tiene la responsabilidad de fortalecer las capacidades y potencialidades de la ciudadanía, de allí que la formación de profesionales demanda un cambio que se ajuste a los requerimientos del siglo XXI, los adelantos científicos, el pensamiento universal, con enfoques y perspectivas renovadas que articulen lo nuevo y el contexto en el que se ubica la universidad, UNESCO (2000). De ahí que, en la última década, la Educación Superior en el Ecuador esté experimentando cambios en las concepciones psicopedagógicas que asume, lo que exige del docente una reflexión profunda de su actuar cotidiano y una mejora eficiente del proceso enseñanza aprendizaje de acuerdo con el tiempo, espacio y lugar que le ha tocado vivir.

Por ello el país centra sus esfuerzos por lograr una educación de calidad a lo largo de la vida que garantice la igualdad, la equidad y la inclusión, constituyendo el derecho a la educación, un componente esencial del Buen Vivir. Según Delors (1996):

La educación a lo largo de la vida conduce directamente a la noción de sociedad educativa, es decir, una sociedad en la que se ofrecen múltiples posibilidades de aprender, tanto en la escuela como en la vida económica, social y cultural (...) actualizar los conocimientos y las competencias (...) perfeccionar su arte y de aprovechar las experiencias realizadas en las distintas esferas de la vida (...) (pág. 37).

Es decir, la educación es un proceso continuo y permanente, durante toda la vida, y debe ser desarrollada de forma participativa, no sólo en lo académico, sino en todas las esferas de actuación del individuo. Muchos autores abordan este proceso desde los términos educación / formación continua / permanente, pero en función de un mismo objetivo, la elevación 
constante de la profesionalidad docente para la mejora del desempeño.

\section{Estado del arte - Antecedentes}

En este marco de nuevas miradas a la formación profesional, la educación permanente es definida por (Fullat, 1975): como una educación integral que abarca toda la vida y todas las posibilidades del ser humano. Por su parte, (Ludosjoski, 1981): la identifica con el perfeccionamiento integral y sin solución de continuidad de la personalidad humana, desde su nacimiento hasta su muerte; mientras (Fonseca, 1984a): la considera el principio pedagógico por medio del cual se indica que el proceso educativo es continuo a lo largo de la vida de los individuos y en todas las circunstancias. Supone acciones constantes de capacitación, actualización y perfeccionamiento.

Fonseca, (1984b): asegura que la educación permanente no es en sí misma una modalidad educativa, por lo contrario, es un vaso comunicante y constituye un principio rector que orienta al sistema educativo y lo impulsa a la búsqueda de nuevas alternativas o recursos pedagógicos que permitan ofrecer formación al hombre según sus diversas etapas evolutivas.

El Ministerio de Educación del Ecuador aborda este tema desde la formación continua y plantea que:

Se parte de la premisa de que el aprendizaje no es estático, por tanto, requiere de un proceso permanente de fortalecimiento de las competencias profesionales y de enriquecimiento de sus prácticas pedagógicas, para alcanzar los estándares de calidad que buscamos en la educación ecuatoriana. (Dirección Nacional de Formación Continua, 2015, pág. 1).

La formación continua para docentes en Ecuador constituyó objeto de atención directa del Ministerio de Educación hasta finales del siglo pasado, desde donde se diseñaban e impartían cursos de capacitación en aras de superar al personal docentes de los diferentes niveles educacionales. En este mismo período, las universidades asumieron algunas responsabilidades 
formativas, en particular con la organización de cursos de diplomado, especialización y maestría. Aunque sin un proceso de planificación nacional.

En el contexto de la Universidad Nacional de Educación, se asume el término educación continua, como una de las formas que ha ido ganando espacio en la reconceptualización del papel de la universidad, entendida por (Fonseca, 1984c): como el conjunto de actividades orientadas a extraer de las experiencias cotidianas nuevos elementos formativos que habiliten a los individuos para la realización de tareas y funciones acordes con el devenir social, y las necesidades de su formación.

Esto implica que adquiera cada día una mayor relevancia el diseño de programas dirigidos a elevar la competencia profesional de los docentes que se desempeñan en las instituciones educativas de todo el país, de ampliar actualizar, renovar conocimientos, habilidades, actitudes, valores, emociones, ya adquiridos, en pos de mejorar perfeccionar las competencias que constituyen nuevas demandas del mercado laboral y profesional. Esto implica un tránsito de una escuela tradicional a una innovadora, que responda a las necesidades de su contexto, en la que el diálogo y la reflexión se constituyan en vías indispensables para la comprensión por parte de los miembros de la comunidad educativa del protagonismo de sus estudiantes y por ende se alcancen niveles superiores de desarrollo en esta esfera de gran significación social.

En este análisis resultaron de interés lo relativo a las experiencias educativas mediadas por las tecnologías desde la Unidad de Estudios de Postgrado, la formación docente continua y factores asociados a la política educativa, el postgrado en el Ecuador y su articulación en el Sistema Nacional de Ciencia y Tecnología, así como los mecanismos de aseguramiento de su calidad. A esto se añade también, se haya hecho alusión a la necesidad de orientar los cambios en el postgrado para promover la adopción de modelos pedagógicos que estimulen la creatividad y la innovación, para convertir al 
alumno en el principal protagonista del proceso enseñanza-aprendizaje

Por ende, la búsqueda de cómo afrontar la educación continua en la UNAE a partir de las problemáticas que históricamente había caracterizado este proceso, se convertía en un reto epistemológico en su proceso de implementación. Promover aprendizajes que fomenten el análisis y la reflexión crítica de la propia práctica del docente para transformarla en relación estrecha con la teoría que lo sustenta, implica la generación de epistemes para lograr un posicionamiento epistemológico en la construcción del conocimiento, la comprensión y la construcción gradual de los saberes.

Es importante destacar que los Programas de Educación Continua que se implementen deben evidenciar la complejidad de este proceso desde una praxis colaborativa e individual, desde la transcripción de la representación de cada realidad. De ahí que debe implementarse desde talleres socializadores que estén centrados en un proceso de diálogo y reflexión sobre problemáticas relacionadas con la educación en el contexto.

La generación de una metodología para la identificación desde fuentes primarias de las necesidades de los docentes, que parta de los problemas profesionales de la práctica pedagógica, de sus vivencias, experiencias y que promuevan desde el trabajo colaborativo y la reflexión, análisis de su propia práctica, constituye una prioridad por la Coordinación de Educación Continua de la UNAE que favorezca no solo, la identificación de las debilidades, sino las fortalezas en la dirección del proceso enseñanza y aprendizaje que realizan, con vista a actualizar y lograr cerrar las brechas de conocimiento en el campo pedagógico y didáctico en los contextos educativos.

Actualizar a los docentes en los fundamentos pedagógicos y didácticos que sustentan una dirección de enseñanza y aprendizaje de excelencia a partir de la indagación reflexiva de las problemáticas educativas de su desempeño profesional, desde un enfoque sistémico e interactivo, y teniendo como premisa la problematización de su práctica educativa a través de la reflexión 
crítica de la actividad pedagógica constituye un objetivo básico de la Educación Continua en la UNAE.

Las instituciones escolares donde laboran los docentes deberán constituir los escenarios de aprendizajes que contribuirán a la transformación de las instituciones educativas, que implica la presencia de un colectivo motivado a afrontar situaciones complejas desde la investigación, que compartan intereses, preocupaciones y búsqueda de soluciones a partir de los problemas de su práctica pedagógica. Un colectivo comprometido en la comprensión del conocimiento y la puesta en práctica de ideales y valores, que indaguen, reflexionen, con una visión integrada del conocimiento que conlleve a la diversificación de actividades en el proceso enseñanza y aprendizaje.

\section{Lineamientos}

Teniendo en cuenta estos argumentos, se declaran como lineamientos a tener en cuenta para la implementación de los programas de educación continua por la UNAE, los siguientes:

1. Provocar la integración entre las instituciones educativas, la universidad y las carreras afines para propiciar la identificación de los problemas de la práctica profesional y su inserción en los programas de educación continua, en el que predomine la motivación y comunicación como vía para potenciar el liderazgo y la toma de decisiones por consenso.

2. Se conforman en la dinámica de indagación y transformación, reconocidas como fuerzas o factores que interactúan en las relaciones interpersonales, teniendo en cuenta los factores individuales, constituidos por la personalidad de cada individuo participante, sus experiencias previas, su ideología, los valores, sus expectativas y necesidades, su motivación, así como la historia y el modo de constitución del grupo mismo, las interacciones, subgrupos y relaciones con otros grupos. 
3. Estimular procesos de cooperación académica entre la universidad e instituciones educativas, sobre la base del reconocimiento de la necesidad del desarrollo de valores.

Es importante retomar en este caso, el rol del docente en el proceso enseñanza - aprendizaje, pues a través de su preparación pedagógica y científica logra extender fuera de sí, todos los elementos que permitan el despliegue del proceso el redescubrimiento y reconstrucción del conocimiento por parte del estudiante; de sus particularidades personales, la relación de comunicación en sus distintos tipos de función (informativa, afectiva y reguladora) que permita un ambiente de cooperación y de colaboración, de actividad conjunta dentro del aula.

El docente tutor tiene que caracterizarse por la autenticidad, seguridad, el respeto y la motivación hacia la actividad; con una adecuada acción generadora de bienestar emocional en los participantes, de interacción, de intercambios, y en los marcos de un clima de respeto en el aula.

4. La elaboración de proyectos de vida para identificar los problemas profesionales y contenidos a desarrollar en los programas de educación continua, desde la reflexión en el trabajo en grupo.

Se asume como proyecto de vida, "la medida en que asumimos conscientemente nuestros ideales y planificamos nuestro camino personal para llegar a ellos. Será por tanto una elevada expresión de nuestra conciencia y un producto del desarrollo de nuestra personalidad". (Pino, s.f.). El propio autor plantea que la posibilidad de crear proyectos y planes de vida eficientes está estrechamente vinculada al conocimiento de sí y del entorno que alcance el sujeto, a la calidad de su autorreflexión y al nivel de autodeterminación desarrollado.

Se desarrolla la elaboración de los proyectos de vida en integración con la del acompañamiento, el trabajo autónomo y los talleres de autorreflexión participativo, lo que facilita identificar las necesidades y potencialidades desde 
el trabajo en grupo. En el caso del taller de autorreflexión participativo, se parte desde el compromiso individual, en el cual se crea un ambiente colaborativo de los participantes de manera que se estimule y propicie el diálogo y la reflexión grupal, en función del cambio cuya fuerza fundamental es la actitud frente a los problemas que deben ser superados y que implican a su vez un crecimiento personal y grupal de todos los participantes. También es importante valorar como resultado de la identificación de las necesidades y potencialidades, los problemas profesionales a los que se enfrentarán los miembros de la comunidad educativa participante.

Reflexionar durante la acción consiste en preguntarse lo que pasa o va a pasar, lo que podemos hacer, lo que hay que hacer, cuál es la mejor táctica, qué orientaciones y qué precauciones hay que tomar, qué riesgos existen, etc. [...] Reflexionar sobre la acción... eso es otra cosa. Es tomar la propia acción como objeto de reflexión, ya sea para compararla con un modelo prescriptivo, a lo que habríamos podido o debido hacer de más o a lo que otro practicante habría hecho, ya sea para explicarlo o hacer una crítica (Perreneud, 2010, págs. 30-31).

Así, la reflexión sobre la acción permite valorar lo realizado en la escuela frente a lo prescrito para definir su viabilidad al favorecer la reorientación de las propias acciones de modo justificado a partir de la observación de lo ocurrido y estimular el desarrollo de la metacognición cuando se es consciente de lo efectuado y de lo que ha incidido en ello.

Lo expuesto ha dado lugar a la consideración de la necesaria presencia de este tipo de ejercicio en la educación superior, pues la reflexión y la práctica reflexiva promueven el potencial de un aprendizaje profundo y significativo. De acuerdo con Brockbank y McGill (2002): el conocimiento socioeducativo sólo llega a tener un significado interiorizado y real cuando el participante del proceso transformador aplica ese saber a sí mismo y lo relaciona, de alguna manera, con su experiencia. Cuando se han generado las condiciones para el 
ejercicio reflexivo, se favorece un tipo de aprendizaje esencial en la educación continua, quien aprende es el principal protagonista de su proceso de aprendizaje, pues encuentra significados para el saber que se genera en sus experiencias a partir de las actividades que lo favorecen y en su interacción con tutores y otros participantes.

5. Enrumbar la Educación Continua desde la investigación acción participativa.

Metodología que requiere la participación de grupos para la indagación y el diálogo, en el que la reflexión colectiva de los participantes se desarrolló en función de lograr la trasformación de la educación en la región. La investigación acción participativa, tiene el objetivo de mejorar y aprender de la propia experiencia desde la reflexión - acción, en la que la participación del grupo y el compromiso ético sustentan todo el proceso, con un enfoque que cumpla los rasgos que caracterizan cada uno de sus términos: investigación, acción y participación. Por ende, debe prevalecer la reflexión, la sistematicidad en la ejecución de las tareas a realizar, el control y la crítica de saberes y experiencias aportando nuevos conocimientos y formas de comprender los problemas del contexto a enfrentar.

En los marcos de las observaciones anteriores Stenhouse, citado por Gómez y Salinas (2011), referencia que el entorno socioeducativo se ve enmarcado por la:

Triada que plantea la naturaleza del conocimiento, naturaleza de la enseñanza, naturaleza del aprendizaje, evidencia una relación fuerte entre epistemología, didáctica y curriculum situando el aula como el escenario social donde se colocan a prueba las hipótesis que guían y desafían la acción de los docentes investigadores (pág. 3).

Esta triada se visualiza en el desarrollo de la Investigación, Educación y la Acción cuya finalidad es enseñar a la gente a descubrir su propio potencial para actuar, liberándoles de estados de dependencia y pasividad previos, y 
llevarlos a comprender que la solución está en el esfuerzo colectivo que ellos mismos puedan tomar para cambiar el estado de cosas, que va desde el diagnostico hasta la transformación de la concepción práctica y crítica de la Educación Continua.

6. Integración del proceso de planificación, organización, regulación, control y evaluación de los procesos a ejecutar.

Establecer interrelaciones entre las potencialidades (fortalezas y debilidades) de los recursos humanos, históricos, sociales y económicos como elementos de integración a los contenidos que son abordados en los programas de educación continua, con un marcado enfoque sistémico.

7. Establecer los acompañamientos en los propios escenarios de aprendizaje, que contribuirán desde la colaboración, el trabajo en grupo, y el respeto a la diversidad de criterios, enrumbar el camino para la construcción del conocimiento con un enfoque interdisciplinar.

En la docencia asistida los talleres socializadores deben favorecer el análisis y la reflexión, pero también la reorganización de la acción del docente tutor desde la retroalimentación. Con la socialización de cada grupo, y las valoraciones que se realicen, se hace imprescindible organizar el tiempo establecido para lograr la lógica en su totalidad, sin barreras que rompan las dinámicas de participación.

La evaluación será formativa, la cual parte del diagnóstico, destinado a identificar debilidades y fortalezas individuales y colectivas, lo que favorecerá la retroalimentación del proceso. Se implementará la autoevaluación, coevaluación y heteroevaluación, teniendo en cuenta los criterios establecidos para ello y desde el trabajo grupal, los espacios de reflexión y el portafolio de registros anecdóticos que facilite la descripción de las reflexiones que se realicen en los diferentes contextos donde intervendrá en grupo.

Es importante destacar que el desarrollo de la relación práctica - teoría - práctica, se hará desde los problemas que cada grupo identificó. El análisis 
de los diferentes contenidos, la búsqueda y valoración de la teoría se irá propiciando desde un enfoque integral, teniendo en cuenta la relación que puede darse entre las categorías que se identifiquen como problemas.

8. La educación continua desde la formación de centro y/o comunidades de prácticas.

El término comunidad de práctica fue acuñado por Wenger (1998): en su labor de observar y analizar el conocimiento que se difunde desde una comunidad científica y buscando potenciar este aspecto a nivel corporativo como una institucionalización de la antigua «tormenta o lluvia de ideas» o de intercambios informales. La finalidad de una comunidad de práctica es la de hacer explícita la transferencia informal de conocimiento, ofreciendo una estructura formal que permite adquirir más conocimiento a través de las experiencias socializadas dentro del grupo, de acuerdo con el programa de capacitación de la Educación Continua. Por esta razón, la propia identidad del grupo se refuerza al potenciar el aprendizaje como un proceso de participación y liderazgo compartido; facilitado por el intercambio de experiencias y la motivación.

La comunidad de práctica no es una comunidad científica como tal, ya que su planteamiento no es la ciencia sino la experiencia de la práctica y la gestión compartida del conocimiento. Esta gestión del conocimiento se realiza siempre de una forma colaborativa y en un proceso continuo de establecer estrategias de participación, liderazgo, identidad y aprovechamiento del conocimiento.

La comunidad de práctica al igual que cualquier grupo de trabajo colaborativo tiene sus propias características que en el caso de la UNAE en el desarrollo de la Educación Continua la definen y caracterizan como una comunidad, respondiendo a quiénes se asocian, para qué lo hacen, con que motivación, en qué ámbito o contexto y cuál es la naturaleza de esta relación, constituyen criterios que posibilitan analizar el sentido y los propósitos de estas 
formas de trabajo colaborativo.

\section{Diseño metodológico}

En consecuencia, las fases para el funcionamiento de la Educación Continua se resumen en:

Fase 1: Acercamiento al contexto. Identificación de necesidades de la comunidad educativa.

- Reconocimiento de los resultados en el Sistema Educativo del contexto desde indagaciones iniciales.

- Identificación de antecedentes de la Educación Superior en el contexto.

- Reconocimiento de las principales problemáticas relacionadas con el impacto de la educación superior en la región.

Fase 2: Diagnóstico al territorio desde espacios de reflexión.

- Creación de espacios de reflexión en la escuela y en la comunidad en función del intercambio de experiencias docentes relevantes y la determinación de nuevas necesidades de EC. Trabajar desde la identificación de grupos focales.

- Elaboración de la metodología para la implementación de los talleres de autorreflexión con la comunidad educativa.

- Exploración del territorio para la recolección de información relevante sobre la educación en la provincia y el cantón desde los grupos focales Recolección y procesamiento de información de los talleres de autorreflexión.

- Difusión de la oferta académica desde la atención personalizada.

- Gestión de los procesos: el análisis de las funciones, recursos, determinación de la participación, distribución de las funciones y cronogramación. 
Fase 3: Diseño e implementación de los procesos a ejecutar.

- Implementación de programa de Educación Continua.

- Implementación de la Lesson Study entre los docentes que participan en el programa.

- Monitoreo de las acciones derivadas de la EC en la escuela en la comunidad y en la Universidad.

Fase 4: Presentación de resultados parciales.

- Resultados derivados del proceso de investigación.

- Resultados de la implementación de la Educación Continua.

Fase 5: Estudio de impacto. Se encuentra en organización.

- Desarrollo de talleres científicos para la divulgación de nuevas experiencias derivadas de la EC y rediseño de la estrategia de EC.

A manera de resumen se representa el modelo integrador e inclusivo de Educación Continua de la UNAE y su implementación en coordinación con el MinEduc y Red de Maestros. 
Figura 1. Modelo integrador e inclusivo de Educación Continua de la UNAE y su implementación en coordinación con el MinEduc y Red de Maestros.

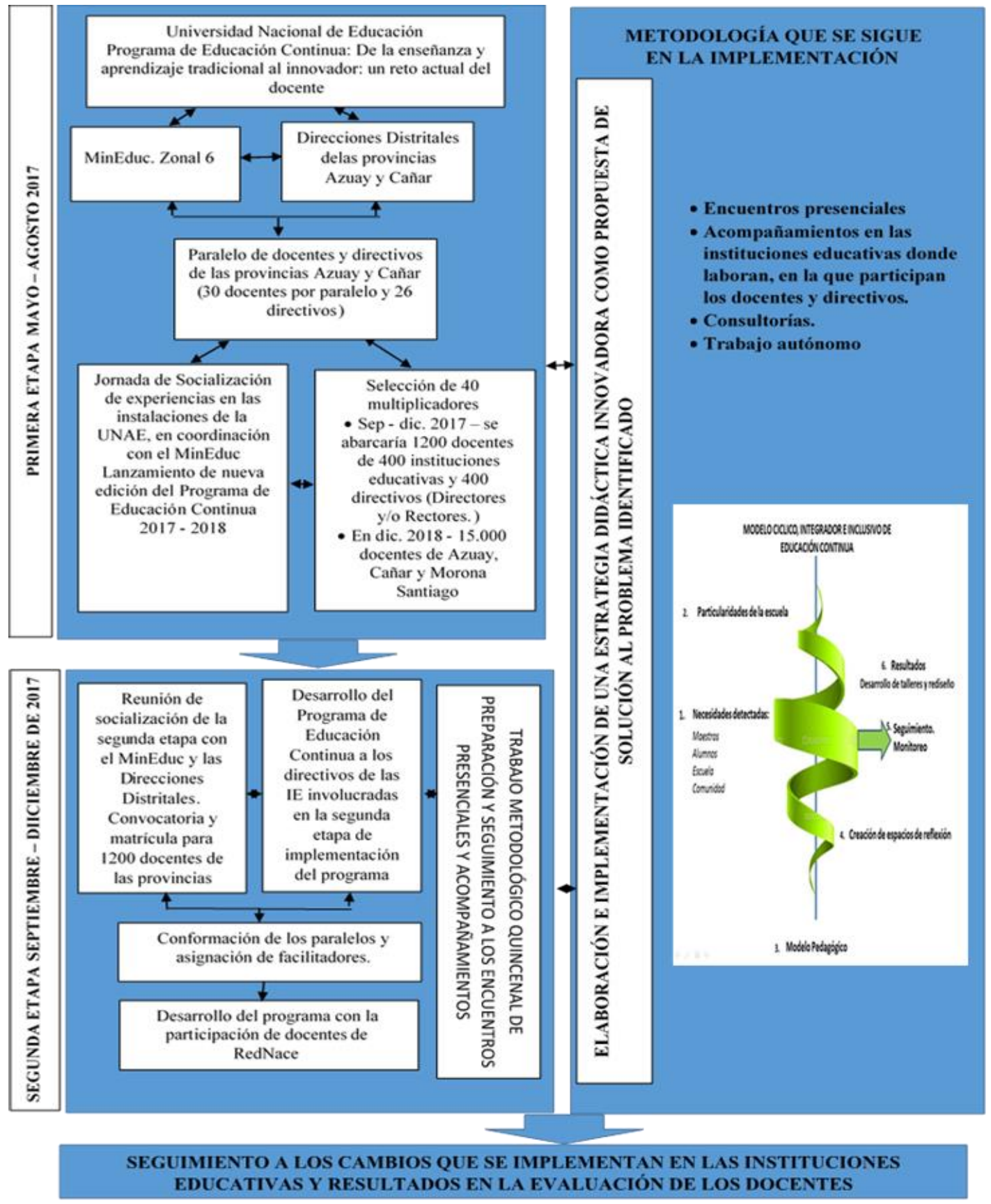

Fuente: Los Autores (2017). 
Cumplir con las fases del modelo y etapas declaradas, implica aprender a identificar las barreras que constituyan resistencia humana a los cambios, lo que propiciará I disminución de la inercia y frustración de los esfuerzos que se invertirán para llevarla a cabo. Identificar las necesidades desde el diagnóstico, así como las fortalezas de los docentes que participan será lo que propiciará el cambio que se necesita para impulsar un programa desde un modelo innovador que respete la individualidad y cooperación, en el que las experiencias de la práctica pedagógica implementada por ellos en las instituciones escolares donde laboran se constituyan en premisas para la identificación de los temas a desarrollar.

\section{Conclusiones}

La premisa fundamental en la Educación Continua en la UNAE lo constituyen las exigencias que establece el modelo pedagógico de la Universidad Nacional de Educación, única en el Ecuador, y que tiene la gran misión de "contribuir a la formación de talento humano del sistema educativo para la construcción de una sociedad justa, equitativa, libre y democrática generando modelos educativos de excelencia caracterizados por rigor científico, enfoque de derechos y de interculturalidad". (UNAE, 2014, pág. s.n.). Además, la idea de que "dos hechos rodean y atraviesan el origen de la UNAE, el cambio de época, y la importancia mundial que tiene la formación de maestros para la transformación de la educación en cualquier país y en concreto en el Ecuador" (Álvarez, Quinn, Pérez, Prats, Didirksso, Peñafiel y Fernández, 2015, pág. 12).

\section{Referencias}

Álvarez, F., Quinn, H., Pérez, Á., Prats, J., Didirksso, A., Peñafiel, F., \& Fernández, S. (2015). Hacer bien, pensar bien y sentir bien, pág. 223. Azogues, Ecuador: Universidad Nacional de Educación. 
Brockbank, A., \& McGill I. (2002). Aprendizaje reflexivo en la educación superior. 2a edición. ISBN: 9788471124524, págs. 312. Madrid, España: Ediciones Morata.

Delors, J. (1996). Compendio. La educación encierra un tesoro. Francia: Ediciones UNESCO, pág. 37.

Dirección Nacional de Formación Continua (2015). Proyecto Sistema Integral de Desarrollo Profesional. Ecuador: Ministerio de Educación. Recuperado de:

https://educacion.gob.ec/wpcontent/uploads/downloads/2015/11/Proyecto-Siprofe.pdf

Freire, P. (1997). A la sombra de este árbol. Primera edición. Barcelona, España: El Roure Editorial, S.A., ISBN: 84-7976-013-3, págs. 170.

Fonseca, L. (1984a,b,c). Educación y Formación Continuada. Caracas: Ediciones UCV: Facultad de Ciencias Económicas y Sociales.

Ludosjoski, R. (1981). Andragogía, educación del adulto. Buenos Aires: Guadalupe.

Perreneud, P. (2010). Desarrollar la práctica reflexiva en el oficio de enseñar. Barcelona, España: Editorial Graó.

Pino, J. (s.f.). El desafío de proyectar la vida. La Habana. Cuba: Universidad de Ciencias Pedagógicas "Enrique José Varona".

Gómez, L., \& Salinas, M. (2011). El currículo como proceso la investigación-acción como método referente esenciales para la formación docente en Colombia. Salamanca, España: Universidad de Salamanca. Recuperado de:

https://es.slideshare.net/mariaeugeniasalinas/el-curriculo-comoproceso-la-ia-como-mtodo-ejes-en-la-formacin-docente-en-colombia Fullat, O. (1975). La Educación Permanente (Biblioteca Salvat de grandes temas; 72 (Spanish Edition). ISBN 10: 8434574306; ISBN 13: 9788434574304. Barcelona, España: Salvat Editores, 
UNAE (2014). Modelo Pedagógico. Azogues, Ecuador: Universidad Nacional de Educación.

UNESCO (2000). Desafíos de la educación. IIPE Buenos Aires. Instituto Internacional de Planeamiento de la Educación. Argentina: Ministerio de Educación de la Nación. Recuperado de:

http://unesdoc.unesco.org/images/0015/001591/159155s.pdf

Wenger, E. (1998). Communities of practice and social learning systems: the career of a concept. New York: Cambridge University Press Recuperado de:

https://wenger-trayner.com/wp-content/uploads/2012/01/09-10-27-

CoPs-and-systems-v2.01.pdf 


\section{Oscar Antonio Martínez Molina \\ e-mail: oscar.martinez@unae.edu.ec}

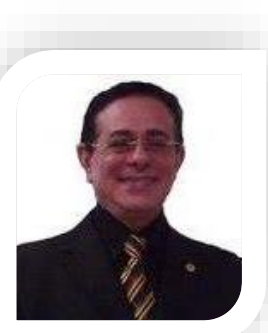

Nacido es San Cristóbal, estado Táchira, Venezuela. Licenciado en Educación Mención Orientación Educativa y Profesional (Universidad de Los Andes, Extensión Táchira). Magíster en Ciencias de la Educación Superior, Mención Andragogía (UNELLEZ). Doctor en Educación Mención Suma Cum Laude (Universidad de Málaga, España), Postdoctor en Estudios Libres de la Universidad Fermín Toro. Diplomado en Educación Abierta y a distancia (Universidad Fermín Toro). Maestría Experto avanzado en E-learning en la Fundación para la actualización tecnológica de Latinoamérica (FATLA). Maestría Experto en Tecnología Educativa nivel avanzado en la Fundación para la actualización tecnológica de Latinoamérica (FATLA). Profesor Jubilado de la Universidad Nacional Abierta. Categoría Académica de Titular. Director de tesis doctorales y de maestría. Responsable de la línea en enseñanzaaprendizaje de investigación del COBAIND. Representante del convenio Editorial Académica Española-COBAIND. Coordinador de la línea de investigación en Educación en la UNEFA Barinas. Presidente/Fundador del Consejo Barinés de Investigación y Desarrollo. Profesor investigador del Programa de Estímulo a la Innovación e Investigación en categoría "A-2". Docente investigación de la Universidad Nacional de Ecuador. 


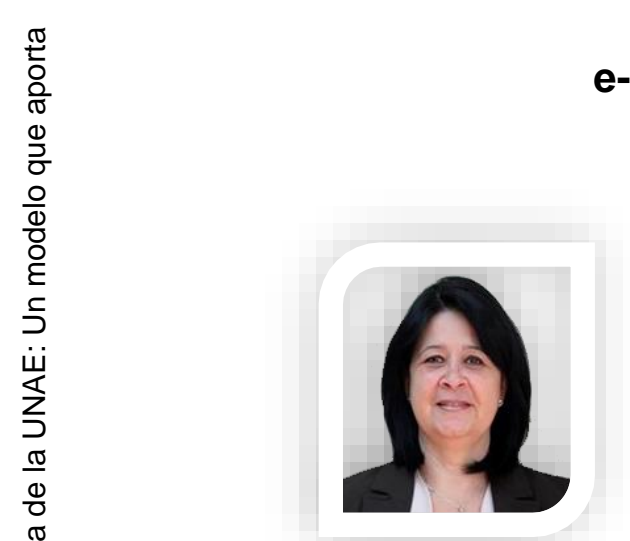

Madelin Rodríguez Rensoli

e-mail: madelin.rodriguez@unae.edu.ec

Nacida en Cuba. Docente Investigadora Titular Principal 1 de la UNAE, Ecuador. Doctora en Ciencias Pedagógicas. Máster en Investigación Educativa. Licenciada en Educación, Especialidad Biología. Acreditada como Investigadora Agregada 1 por la SENESCYT. Ha participado como directora e investigadora en proyectos de investigaciones. Actualmente Directora de Educación Continua y Directora de proyecto de investigación y tesis de maestría y doctorado. Ha publicado en revistas indexadas y en Congresos. Miembro del Consejo Editorial de la Revista Panorama Médico, IPLAC y Gaceta Médica Espirituana. Miembro del Equipo de Árbitros de la Revista Scientific. Ha participado como evaluadora externa en procesos de acreditación Cuba y Ecuador.

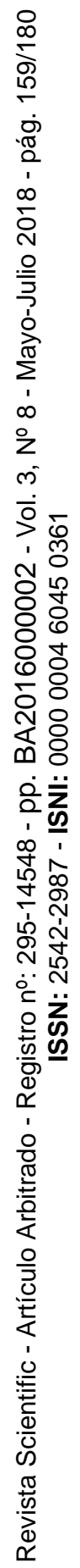

179 


\section{Wilfredo García Felipe \\ e-mail: wilfredo.garcia@unae.edu.ec}

Nacido en Cuba. Docente Investigador Titular Principal

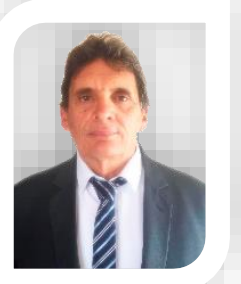

1. Doctor en Ciencias Pedagógicas. Máster en

Educación Avanzada. Licenciado en Ciencias Sociales.

Acreditado como investigador por la SENESCYT en

Ecuador. Actualmente Codirector de Proyecto de Investigación. Director de Carrera y de tesis de Maestrías y Doctorado. Ha cumplido responsabilidades como docente investigador y directivas en diferentes niveles de educación. He publicado artículos derivados de proyectos de investigación en revistas indexadas, así como capítulos de libros. Ha participado en investigación y colaborado en proyectos sobre gestión educacional, en Congresos Internacionales. Director de tesis de doctorado y de maestrías con temas relacionados a la Didáctica y Pedagogía.

El contenido de este manuscrito se difunde bajo una Licencia de Creative Commons ReconocimientoNoComercial-Compartirlgual 4.0 Internacional 